\title{
On 2-fold Completeness of the Eigenfunctions for the Strongly Irregular Quadratic Pencil of Differential Operators of Second Order
}

\section{O. V. Parfilova}

Saratov State Law Academy, Russia, 410056, Saratov, Volskaya st., 1, Oksana_Parfilova@mail.ru

A class of strongly irregular pencils of ordinary differential operators of second order with constant coefficients is considered. The roots of the characteristic equation of the pencils from this class are supposed to lie on a straight line coming through the origin and on the both side of the origin. Exact interval on which the system of eigenfunctions is 2-fold complete in the space of square summable functions is finded.

Key words: quadratic pencil, second order pencil, pencil of ordinary differential operators, two-point boundary conditions, homogeneous differential expression with constant coefficients, completeness of the system of eigenfunctions, non-completeness of the system of eigenfunctions.

\section{References}

1. Shkalikov A. A. Boundary value problems for ordinary differential equations with a parameter in the boundary conditions J. of Math. Sciences, 1986, vol. 33, iss. 6, pp. 1311-1342.

2. Rykhlov V. S. On completeness of eigenfunctions of quadratic penciles of ordinary differential operators. Russian Math. [Izv. VUZ. Matematika], 1992, vol. 36, no. 3, pp. 33-42.

3. Rykhlov V. S. On properties of eigenfunctions of ordinary differential quadratic pencil of the second order. Integral Transforms and Special Functions. Inform. Byulleten, 2001, vol. 2, no. 1, pp. 85-103 (in Russian).

4. Rykhlov V. S. Double completeness of eigenfunctions of a quadratic pencil of second order differential operators.
Zbirnik prats' In-tu matematiki NAN Ukraini, 2009, vol. 6, no. 1, pp. 237-249 (in Russian).

5. Rykhlov V. S. O polnote sobstvennykh funktsii differentsial'nogo puchka vtorogo poriadka, korni kharakteristicheskogo uravneniia kotorogo lezhat na odnoi priamoi [On completeness of eigenfunctions of a differential pencil of the second order the roots of the characteristic equation of which lie on a straight line]. Matematika. Mehanika. Saratov, 2007, iss. 9, pp. 88-91 (in Russian).

6. Rykhlov V. S. On completeness of eigenfunctions for pencils of differential operators. Spectral and Evolutional Problems : Proc. of the Seventh Crimean Autumn Mathematical School-Symposium. Simferopol, 1997, vol. 7, pp. 70-73 (in Russian).

\section{УДК 517.9}

\section{О СТРУКТУРЕ ОПЕРАТОРА, ОБРАТНОГО К ИНТЕГРАЛЬНОМУ ОПЕРАТОРУ СПЕЦИАЛЬНОГО ВИДА}

\section{В. Е. Струков}

Аспирант кафредры математических методов исследования операций, Воронежский государственный университет, sv.post.of.chaos@gmail.com

В статье рассматривается алгебра с единицей, порожденная интегральными операторами, действующими в пространствах непрерывных периодических фрункций. Доказывается наполненность этой подалгебры в алгебре всех линейных ограниченных операторов.

Ключевые слова: банахово пространство, интегральный оператор, теорема Бохнера-Филлипса, ряд Фурье оператора, наполненность подалгебры, винеровская пара алгебр.

Пусть $l^{1}(\mathbb{Z})$ - банахово пространство двусторонних суммируемых последовательностей $a: \mathbb{Z} \rightarrow \mathbb{C}$ с нормой $\|a\|_{1}=\sum_{k \in \mathbb{Z}}|a(k)|<\infty$.

Символом $C(\mathbb{T})$ будем обозначать банахово пространство комплексных непрерывных функций, определенных на окружности $\mathbb{T}=\{\theta \in \mathbb{C}:|\theta|=1\}$.

Будем говорить, что функция $f \in C(\mathbb{T})$ обладает абсолютно сходящимся рядом Фурье, если она может быть представлена в виде ряда $f(\theta)=\sum_{k \in \mathbb{Z}} a(k) \theta^{k}, \theta \in \mathbb{T}$, где $a \in l^{1}(\mathbb{Z})$. Совокупность всех таких функций обозначим через $A C(\mathbb{T})$. Заметим, что $A C(\mathbb{T})$ является банаховой алгеброй с поточечным умножением и нормой

$$
\|f\|_{A C}=\|a\|_{1}=\sum_{k \in \mathbb{Z}}|a(k)| .
$$


В терминах введенных обозначений теорема Винера формулируется следующим образом.

Теорема 1 (Н. Винер). Если функция $f \in A C(\mathbb{T})$ u $f(\theta) \neq 0$ для всех $\theta \in \mathbb{T}$, mо $1 / f \in A C(\mathbb{T})$, m. е. $1 / f(\theta)=\sum_{k \in \mathbb{Z}} b(k) \theta^{k}$, где $b \in l^{1}(\mathbb{Z})$.

Согласно теории И. М. Гельфанда, теорему Винера можно рассматривать на языке пар алгебр как утверждение о том, что алгебры $A C(\mathbb{T})$ и $C(\mathbb{T})$ образуют винеровскую пару [1].

Определение 1. Будем говорить, что алгебры $\mathscr{A}, \mathscr{B}(\mathscr{A} \subset \mathscr{B})$ образуют винеровскую пару, если каждый элемент $a \in \mathscr{A}$, обратимый в алгебре $\mathscr{B}$, обратим также в алгебре $\mathscr{A}$.

В данной работе будет использоваться терминология Н. Бурбаки [2].

Определение 2. Подалгебра $\mathscr{A} \subset \mathscr{B}$ называется наполненной в алгебре $\mathscr{B}$, если каждый элемент $a \in \mathscr{A}$, обратимый в алгебре $\mathscr{B}$, обратим также в подалгебре $\mathscr{A}$.

В терминах наполненности теорема Винера принимает следующий вид.

Теорема 2. Алгебра $A C(\mathbb{T})$ наполнена в алгебре $C(\mathbb{T})$.

Для функций со значениями в банаховой алгебре теорема Винера была получена в статье S. Bochner, R. S. Fillips [3]. На основе результатов этой статьи в работах А. Г. Баскакова [4-7] был развит метод доказательства для элементов из банахова пространства линейных ограниченных операторов. Введем терминологию, взяв за основу статью [8].

Пусть End $X$ - банахова алгебра линейных ограниченных операторов на бесконечномерном комплексном банаховом пространстве $X$. Обозначим через Inv $X$ множество (непрерывно) обратимых операторов из пространства End $X$.

Рассматривается сильно непрерывное изометрическое $2 \pi$-периодическое представление:

$$
T: \mathbb{R} \rightarrow \text { End } X, \quad T(t+2 \pi)=T(t), \quad t \in \mathbb{R} .
$$

Каждому оператору $A \in \operatorname{End} X$ поставим в соответствие периодическую с периодом $2 \pi$ сильно непрерывную функцию $\Phi_{A}: \mathbb{R} \rightarrow$ End $X$, определяемую равенством

$$
\Phi_{A}(t)=T(t) A T(-t), \quad t \in \mathbb{R}
$$

Множество операторов $A \in \operatorname{End} X$, для которых операторнозначная функция $\Phi_{A}$ непрерывна в равномерной операторной топологии, обозначим символом $\operatorname{End}_{c} X$, а подмножество непрерывно обратимых операторов из $\operatorname{End}_{c} X$ обозначим через $\operatorname{Inv}_{c} X$.

Функции $\Phi_{A}$ поставим в соответствие ее ряд Фурье:

$$
\Phi_{A}(t) \sim \sum_{k \in \mathbb{Z}} A_{k} e^{i k t}, \quad t \in \mathbb{R}
$$

где

$$
A_{k}=\frac{1}{2 \pi} \int_{0}^{2 \pi} T(t) A T(-t) e^{-i k t} d t, \quad k \in \mathbb{Z} .
$$

Ряд $\sum_{k \in \mathbb{Z}} A_{k}$ будем называть рядом Фурье оператора $A$, а операторы $A_{k}, k \in \mathbb{Z},-$ коэффициентами Фурье этого оператора (относительно представления $T$ ).

Определим двустороннюю последовательность $d_{A}(k)$, положив $d_{A}(k)=\left\|A_{k}\right\|, k \in \mathbb{Z}$.

Пусть выполнено следующее условие.

Условие 1. Для оператора $A \in \operatorname{End}_{c} X$ справедливо одно из соотношений

1. $\sum_{k \in \mathbb{Z}} d_{A}(k)<\infty$;

2. $\sum_{k \in \mathbb{Z}} d_{A}(k) \alpha(k)<\infty$, где функизия $\alpha: \mathbb{Z} \rightarrow \mathbb{R}_{+}$удовлетворяет следующим свойствам:

(a) $\alpha(k) \geq 1$ для всех $k \in \mathbb{Z}$,

(b) $\alpha\left(k_{1}+k_{2}\right) \leq \alpha\left(k_{1}\right) \alpha\left(k_{2}\right)$ для всех $k_{1}, k_{2} \in \mathbb{Z}$,

(c) $\lim _{n \rightarrow \infty} \frac{\ln \alpha(n k)}{n}=0$ для всех $k \in \mathbb{Z}$;

3. $\lim _{k \rightarrow \infty} d_{A}(k)|k|^{\gamma}=0, k \in \mathbb{Z}, \gamma>1$. 
Совокупности операторов, удовлетворяющих пунктам 1, 2 или 3 условия 1, обозначим соответственно $\operatorname{End}_{1} X, \operatorname{End}_{\alpha} X$ и $\operatorname{End}_{\gamma} X$. Нетрудно показать, что данные классы являются подалгебрами алгебры $\operatorname{End}_{c} X$. Обозначим символом $p$ в $\operatorname{End}_{p} X$ один из символов $1, \alpha, \gamma$. Из лемм 1 и 2 статьи [5] вытекает следующая теорема.

Теорема 3. Пусть оператор $A \in \operatorname{Inv}_{c} X \cap \operatorname{End}_{p} X$. Tогдa $A^{-1} \in \operatorname{End}_{p} X$.

Введем используемые далее обозначения. Символом $L_{2 \pi}^{1}(\mathbb{R}, \mathbb{C})$ обозначим банахову алгебру измеримых $2 \pi-$ периодических, интегрируемых на отрезке длины $2 \pi$ функций $f: \mathbb{R} \rightarrow \mathbb{C}$ с нормой $\|f\|_{1}^{2 \pi}=\frac{1}{2 \pi} \int_{0}^{2 \pi}|f(t)| d t$. В качестве операции умножения возьмем свертку $(f * g)(t)=\frac{1}{2 \pi} \int_{0}^{2 \pi} f(s) g(t-s) d s$, для всех $f, g \in L_{2 \pi}^{1}(\mathbb{R}, \mathbb{C})$.

В данной статье рассматриваются операторы вида

$$
A=a I+K, \quad a \in \mathbb{C} \backslash\{0\}
$$

где $K$ - интегральный оператор, действующий в банаховом пространстве $X=C_{2 \pi}(\mathbb{R}, \mathbb{C})$ непрерывных функций (периода $2 \pi$ ) с нормой $\|x\|_{C_{2 \pi}}=\max _{t \in[0,2 \pi]}|x(t)|$.

Оператор $K$ имеет вид

$$
(K x)(\tau)=\int_{0}^{2 \pi} \mathscr{K}(\tau, u) x(u) d u,
$$

причем его ядро $\mathscr{K}: \mathbb{R} \times \mathbb{R} \rightarrow \mathbb{C}$ обладает следующими свойствами:

1) $\mathscr{K}(\tau+2 \pi, u+2 \pi)=\mathscr{K}(\tau, u)$ для всех $\tau, u \in \mathbb{R}$;

2) $\kappa(\tau) \in L_{2 \pi}^{1}(\mathbb{R}, \mathbb{C})$ для всех $\tau \in \mathbb{R}$, где $(\kappa(\tau))(u)=\mathscr{K}(\tau, u), u \in \mathbb{R}$;

3) $\kappa \in C_{2 \pi}\left(\mathbb{R}, L_{2 \pi}^{1}(\mathbb{R}, \mathbb{C})\right.$ ) (функция $\kappa$ непрерывна по норме пространства $L_{2 \pi}^{1}(\mathbb{R}, \mathbb{C})$ ).

Легко проверить, что рассматриваемый оператор $K$ определен корректно, т. е. $K x \in C_{2 \pi}(\mathbb{R}, \mathbb{C})$ для любого $x \in C_{2 \pi}(\mathbb{R}, \mathbb{C})$.

В качестве группы изометрий в пространстве $C_{2 \pi}(\mathbb{R}, \mathbb{C})$ рассмотрим группу сдвигов $S: \mathbb{R} \rightarrow$ End $C_{2 \pi}(\mathbb{R}, \mathbb{C})$, определенную формулой $(S(t) x)(\tau)=x(\tau+t)$.

Рассмотрим функцию $\Phi_{K}: \mathbb{R} \rightarrow$ End $C_{2 \pi}(\mathbb{R}, \mathbb{C})$, определяемую формулой $\Phi_{K}(t)=S(t) K S(-t)$. Ясно, что она имеет вид

$$
\left(\Phi_{K}(t) x\right)(\tau)=\int_{0}^{2 \pi} \mathscr{K}(\tau+t, v+t) x(v) d v, \quad x \in C_{2 \pi}(\mathbb{R}, \mathbb{C}) .
$$

Пусть оператор вида (1) удовлетворяет одному из пунктов условия 1. Обозначим соответствующие совокупности операторов символами $\operatorname{Int}_{1} C_{2 \pi}(\mathbb{R}, \mathbb{C}), \operatorname{Int}_{\alpha} C_{2 \pi}(\mathbb{R}, \mathbb{C}), \operatorname{Int}_{\gamma} C_{2 \pi}(\mathbb{R}, \mathbb{C})$. Несложно показать, что данные совокупности являются банаховыми подалгебрами алгебры $\operatorname{End} C_{2 \pi}(\mathbb{R}, \mathbb{C})$.

Лемма 1. Рассматриваемьй оператор $K \in \operatorname{End} C_{2 \pi}(\mathbb{R}, \mathbb{C})$ компактен.

Доказательство. Докажем данное утверждение с помощью теоремы Арцела, т. е. покажем, что оператор $K$ отображает единичный шар $B(0,1) \subset$ End $C_{2 \pi}(\mathbb{R}, \mathbb{C})$ в равномерно ограниченное и равностепенно непрерывное множество. В силу оценки

$$
\|K x\|=\max _{t \in[0,2 \pi]}\left|\int_{0}^{2 \pi} \mathscr{K}(t, s) x(s) d s\right| \leq \max _{t \in[0,2 \pi]}\|\kappa(t)\|_{1}^{2 \pi} \equiv \text { const }
$$

для всех $x \in B(0,1)$, где $(\kappa(t))(s)=\mathscr{K}(t, s), t, s \in \mathbb{R}$, получаем, что множество $K B(0,1)$ равномерно ограниченно. Для всех $x \in B(0,1)$ из соотношения

$$
\begin{gathered}
|(K x)(t)-(K x)(t+\tau)|=\left|\int_{0}^{2 \pi} \mathscr{K}(t, s) x(s) d s-\int_{0}^{2 \pi} \mathscr{K}(t+\tau, s) x(s) d s\right| \leq \\
\leq\|x\|_{C_{2} \pi} \int_{0}^{2 \pi}|\mathscr{K}(t, s)-\mathscr{K}(t+\tau, s)| d s \leq\|\kappa(t)-\kappa(t+\tau)\|_{1}^{2 \pi},
\end{gathered}
$$

а также из того, что $\kappa \in C_{2 \pi}\left(\mathbb{R}, L_{2 \pi}^{1}(\mathbb{R}, \mathbb{C})\right)$, следует, что множество $K B(0,1)$ является равностепенно непрерывным. 
Лемма 2. Рассмотрим в пространстве $L_{2 \pi}^{1}$ оператор сдвига $S: \mathbb{R} \rightarrow \operatorname{End} L_{2 \pi}^{1}(\mathbb{R}, \mathbb{C})$. Совокупность функций $\{S(\tau)(\mathscr{K}(t, s))\}=\{\mathscr{K}(t, \tau+s), \tau, t, s \in \mathbb{R}\}$ равностепенно непрерывна по норме пространства $L_{2 \pi}^{1}(\mathbb{R}, \mathbb{C})$ относительно переменной $\tau$.

Доказательство. Для доказательства данного факта достаточно показать равностепенную непрерывность при $\tau=0$. Зафиксируем $\varepsilon>0$. Сначала докажем, что семейство функций $\{\kappa(t), t \in \mathbb{R}\}=$ $=\{\mathscr{K}(t, \cdot), t \in \mathbb{R}\}$ является предкомпактным множеством в $L_{2 \pi}^{1}(\mathbb{R}, \mathbb{C})$. Поскольку функция $\kappa$ непрерывна по норме пространства $L_{2 \pi}^{1}(\mathbb{R}, \mathbb{C})$ и периодична, то она равномерно непрерывна по норме пространства $L_{2 \pi}^{1}(\mathbb{R}, \mathbb{C})$, в силу чего выберем $\delta_{1}>0$ так, чтобы при всех $t_{1}, t_{2} \in \mathbb{R}$ из того, что $\left|t_{1}-t_{2}\right|<\delta_{1}$, всегда следовало, что $\left\|\kappa\left(t_{1}\right)-\kappa\left(t_{2}\right)\right\|_{1}^{2 \pi}<\varepsilon / 3$.

В силу периодичности функции $\kappa$ с помощью разбиения $\left\{t_{k}\right\}_{k=0}^{n}$ отрезка $[0,2 \pi]$ с диаметром $\delta_{1}$ можно построить конечную $\varepsilon / 3$-сеть $\left\{\kappa\left(t_{k}\right)\right\}_{k=0}^{n}$ для множества функций $\{\kappa(t), t \in \mathbb{R}\} \subset L_{2 \pi}^{1}(\mathbb{R}, \mathbb{C})$.

Поскольку множество непрерывных функций плотно в $L_{2 \pi}^{1}[9]$, для каждой функции $f \in L_{2 \pi}^{1}(\mathbb{R}, \mathbb{C})$ можно подобрать последовательность непрерывных периодических функций $\left\{f_{m}\right\} \subset C_{2 \pi}(\mathbb{R}, \mathbb{C})$, такую, что $\left\|f-f_{m}\right\|_{1}^{2 \pi} \rightarrow 0$ при $m \rightarrow \infty$. Для величины $\varepsilon / 9$ выберем такое $m_{0}=m_{0}(\varepsilon / 9, f)$, что для всех $m>m_{0}\left\|f-f_{m}\right\|_{1}^{2 \pi} \leq \varepsilon / 9$. В силу равномерной непрерывности периодической функции $f_{m_{0}}$ выберем такое $\delta=\delta\left(\varepsilon / 9, f_{m_{0}}\right)>0$, что для всех $\tau \in \mathbb{R},|\tau|<\delta$, справедливо, что $\left\|f_{m_{0}}-S(\tau) f_{m_{0}}\right\|_{1}^{2 \pi} \leq \varepsilon / 9$. Таким образом, для произвольной функции $f \in L_{2 \pi}^{1}(\mathbb{R}, \mathbb{C})$ и произвольного числа $\tau \in \mathbb{R},|\tau|<\delta$, из соотношения

$$
\|f-S(\tau) f\|_{1}^{2 \pi} \leq\left\|f-f_{m_{0}}\right\|_{1}^{2 \pi}+\left\|f_{m_{0}}-S(\tau) f_{m_{0}}\right\|_{1}^{2 \pi}+\left\|S(\tau) f_{m_{0}}-S(\tau) f\right\|_{1}^{2 \pi}
$$

следует, что $\|f-S(\tau) f\|_{1}^{2 \pi} \leq \varepsilon / 3$.

Теперь для каждой функции из указанной выше конечной $\varepsilon / 3$-сети $\left\{\kappa\left(t_{k}\right)\right\}_{k=0}^{n}$ найдем указанным выше образом величины и функции $m_{0}\left(\varepsilon / 9, \kappa\left(t_{k}\right)\right), f_{m_{0}\left(\varepsilon / 9, \kappa\left(t_{k}\right)\right)}, \delta\left(\varepsilon / 6, f_{m_{0}\left(\varepsilon / 9, \kappa\left(t_{k}\right)\right)}\right)$ и выберем

$$
\delta_{2}=\min _{0 \leq k \leq n}\left\{\delta\left(\varepsilon / 9, f_{m_{0}\left(\varepsilon / 9, \kappa\left(t_{k}\right)\right)}\right)\right\}>0 .
$$

При этом из условия $|\tau|<\delta_{2}$ для всех $k=\overline{0, n}$ будет следовать, что $\left\|\kappa\left(t_{k}\right)-S(\tau) \kappa\left(t_{k}\right)\right\| \leq \varepsilon / 3$.

Фиксируем произвольное $t \in[0,2 \pi]$, выберем соответствующее $t_{k_{0}} \in\left\{t_{k}\right\}_{k=0}^{n}$ такое, что $\left\|\kappa(t)-\kappa\left(t_{k}\right)\right\|_{1}^{2 \pi}<\varepsilon / 3$, возьмем также $\delta_{3}=\min \left\{\delta_{1}, \delta_{2}\right\}$ и $|\tau|<\delta_{3}$. Из соотношения

$$
\|\kappa(t)-S(\tau) \kappa(t)\|_{1}^{2 \pi} \leq\left\|\kappa(t)-\kappa\left(t_{k}\right)\right\|_{1}^{2 \pi}+\left\|\kappa\left(t_{k}\right)-S(\tau) \kappa\left(t_{k}\right)\right\|_{1}^{2 \pi}+\left\|S(\tau) \kappa\left(t_{k}\right)-S(\tau) \kappa(t)\right\|_{1}^{2 \pi}
$$

для произвольного $t \in[0,2 \pi]$ и произвольного $|\tau|<\delta_{3}$ получим, что

$$
\|\kappa(t)-S(\tau) \kappa(t)\|_{1}^{2 \pi}<\varepsilon
$$

Лемма 3. Для рассматриваемого оператора $K \in \operatorname{End} C_{2 \pi}(\mathbb{R}, \mathbb{C})$ функция $\Phi_{K}$ непрерьвна в равномерной операторной топологии.

Доказательство. Для доказательства данного факта достаточно проверить непрерывность в нуле.

Фиксируем произвольное $\varepsilon>0$. Справедливо соотношение

$$
\begin{gathered}
\left\|\Phi_{K}(t)-\Phi_{K}(0)\right\|=\|S(t) K S(-t)-K\|= \\
=\sup _{\|x\| \leq 1}\|(S(t) K S(-t)-K) x\|=\sup _{\|x\| \leq 1} \max _{\tau \in[0,2 \pi]}\left\|\int_{0}^{2 \pi}(\mathscr{K}(\tau+t, v+t)-\mathscr{K}(\tau, v)) x(v) d v\right\| \leq \\
\leq \max _{\tau \in[0,2 \pi]} \int_{0}^{2 \pi}|\mathscr{K}(\tau+t, v+t)-\mathscr{K}(\tau, v)| d v=\int_{0}^{2 \pi}|\mathscr{K}(\tau+t, v+t) \pm \mathscr{K}(\tau+t, v)-\mathscr{K}(\tau, v)| d v \leq \\
\leq \int_{0}^{2 \pi}|\mathscr{K}(\tau+t, v)-\mathscr{K}(\tau, v)| d v+\int_{0}^{2 \pi}|\mathscr{K}(\tau+t, v+t)-\mathscr{K}(\tau+t, v)| d v .
\end{gathered}
$$

В силу свойства 3 ядра оператора $K$ можно подобрать такое $\delta_{1}>0$, что при $|t|<\delta_{1}$ первое слагаемое в правой части выражения выше будет меньше, чем $\varepsilon / 2$. В силу леммы 2 можно выбрать величину $\delta_{2}>0$ такую, что для всех $t,|t|<\delta_{2}$, второе слагаемое будет меньшим $\varepsilon / 2$.

Выберем $\delta=\min \left\{\delta_{1}, \delta_{2}\right\}$, тогда для всех $t,|t|<\delta$, будет справедливо

$$
\left\|\Phi_{K}(t)-\Phi_{K}(0)\right\|<\varepsilon \text {. }
$$

т. е. функция $\Phi_{K}$ непрерывна в равномерной операторной топологии. 
Рассмотрим структуру коэффициентов Фурье функции $\Phi_{K}$. Поставим функции $\Phi_{K}$ в соответствие ее ряд Фурье:

$$
\Phi_{K}(t) x \sim \sum_{k \in \mathbb{Z}} K_{n} x e^{i n t}
$$

где коэффициенты Фурье определяются следующими формулами:

$$
\left(K_{n} x\right)(\tau)=\int_{0}^{2 \pi} \mathscr{K}_{n}(\tau, v) x(v) e^{i n v} d v
$$

где

$$
\mathscr{K}_{n}(\tau, v)=\frac{1}{2 \pi} \int_{0}^{2 \pi} \mathscr{K}(\tau+t, v+t) e^{-i n(t+v)} d t
$$

Коэффициенты Фурье оператора $A$ будут совпадать с коэффициентами оператора $K$ за исключением нулевого коэффициента, который будет иметь вид $A_{0}=a I+K_{0}$.

С помощью замены переменных несложно показать, что $\mathscr{K}_{n}(\tau+u, v+u)=\mathscr{K}_{n}(\tau, v)$ для всех $u \in \mathbb{R}$, откуда следует, что $\mathscr{K}_{n}(\tau, v)=\mathscr{K}_{n}(\tau-v, 0)=\mathscr{K}_{n}^{0}(\tau-v)$, т. е. функция $\mathscr{K}_{n}$ на самом деле зависит от разности аргументов.

Через $E_{n} \in \operatorname{End} C_{2 \pi}(\mathbb{R}, \mathbb{C})$ обозначим оператор умножения на экспоненту: $\left(E_{n} x\right)(t)=e^{i n t} x(t)=$ $=\left(e_{n} x\right)(t), n \in \mathbb{Z}, t \in \mathbb{R}$.

Лемма 4. Пусть $A_{n}$ - коэффициенты Фурье некоторого оператора $A \in$ End $C_{2 \pi}(\mathbb{R})$ с непрерывной в равномерной операторной топологии функцией $\Phi_{A}(t)$, тогда операторы $A_{n} E_{-n}$ nерестановочны со сдвигом при всех $n \in \mathbb{Z}$.

Доказательство. Покажем, что $S(\omega) A_{n} S(-\omega)=e^{i n \omega} A_{n}$ при всех $\omega \in \mathbb{R}$ и для любого $n \in \mathbb{Z}$. Рассмотрим произвольное $\omega \in \mathbb{R}$.

$$
\begin{gathered}
S(\omega) A_{n} S(-\omega)=\frac{1}{2 \pi} \int_{0}^{2 \pi} S(\omega) S(t) A S(-t) S(-\omega) e^{-i n t} d t= \\
=\frac{e^{i n \omega}}{2 \pi} \int_{0}^{2 \pi} S(\omega+t) A S(-t-\omega) e^{-i n(t+\omega)} d t=\frac{e^{i n \omega}}{2 \pi} \int_{0}^{2 \pi} S(t) A S(-t) e^{-i n t} d t=e^{i n \omega} A_{n} .
\end{gathered}
$$

Тогда

$$
S(t) A_{n} E_{-n} S(-t)=S(t) A_{n} S(-t)\left(S(t) E_{-n} S(-t)\right)=S(t) A_{n} S(-t) e^{-i n t} E_{-n}=A_{n} E_{-n},
$$

т. е. оператор $A_{n} E_{-n}$ перестановочен со сдвигом.

Так как оператор $A$ непрерывно обратим, то его обратный имеет вид $A^{-1}=\frac{1}{a} I+\widetilde{K}$, где $\widetilde{K}=-\frac{1}{a} K A^{-1}-$ компактный оператор (как произведение компактного и ограниченного).

В силу того что оператор $A \in \operatorname{Int}_{p} C_{2 \pi}(\mathbb{R}, \mathbb{C}) \subset \operatorname{End}_{p} C_{2 \pi}(\mathbb{R}, \mathbb{C}) \subseteq \operatorname{End}_{1} C_{2 \pi}(\mathbb{R}, \mathbb{C})$, где $p-$ один из символов $1, \alpha, \gamma$, по теореме 3 из абсолютной сходимости ряда Фурье функции $\Phi_{A}$ следует абсолютная сходимость рядов Фурье функций $\Phi_{A^{-1}}$ и $\Phi_{\widetilde{K}}$.

Введем понятие аппроксимативной единицы. Для этого возьмем произвольный элемент $x \in C_{2 \pi}(\mathbb{R}, \mathbb{C})$. Его ряд Фурье имеет вид

$$
x(t) \sim \sum_{k \in \mathbb{Z}} x_{k} e_{k} .
$$

Рассмотрим аппроксимативную единицу $\Psi_{n} \in \operatorname{End} C_{2 \pi}(\mathbb{R}, \mathbb{C})$, которая определяется по следующей формуле:

$$
\Psi_{n} x=\sum_{|k| \leq n}\left(1-\frac{|k|}{n}\right) x_{k} e_{k} .
$$

Данная последовательность операторов сильно сходится к тождественному оператору в пространстве End $C_{2 \pi}(\mathbb{R}, \mathbb{C})$, т. е. для всех $x \in C_{2 \pi}(\mathbb{R}, \mathbb{C}) \lim _{n \rightarrow \infty} \Psi_{n} x=x$.

Лемма 5. Для того чтобы оператор $A \in \underset{n \rightarrow \infty}{A \rightarrow} C_{2 \pi}(\mathbb{R}, \mathbb{C})$ был перестановочен со сдвигом, необходимо и достаточно, чтобы функиии е для всех $n \in \mathbb{Z}$, где $\alpha_{n} \in \mathbb{C}$. 
Доказательство. Необходимость. Пусть оператор $A$ перестановочен со сдвигом, т. е. $S(t) A=$ $=A S(t)$ для всех $t \in \mathbb{R}$. Рассмотрим функцию $x_{n}=A e_{n}$ и ее сдвиг $S(h) x_{n}, h \in \mathbb{R}$.

$$
S(h) x_{n}=S(h) A e_{n}=A S(h) e_{n}=A\left(e^{i n h} e_{n}\right)=e^{i n h} A e_{n}=e^{i n h} x_{n} .
$$

Рассмотрим теперь функцию $\widetilde{x_{n}}=x_{n} e_{-n}$ и ее сдвиг $S(h) \widetilde{x_{n}}$.

$$
S(h) \widetilde{x_{n}}=S(h)\left(x_{n} e_{-n}\right)=S(h) x_{n} S(h) e_{-n}=e^{i n h} x_{n} e^{-i n h} e_{-n}=\widetilde{x_{n}} .
$$

Получаем, что $S(h) \widetilde{x_{n}}=\widetilde{x_{n}}$ для всех $h \in \mathbb{R}$, т. е. $\widetilde{x_{n}}-$ постоянная функция.

Обозначим $\widetilde{x_{n}}=x_{n} e_{-n}=\alpha_{n}$, где $\alpha_{n} \in \mathbb{R}$. Тогда $x_{n}=A e_{n}=\alpha_{n} e_{n}$ для всех $n \in \mathbb{Z}$.

Достаточность. Пусть выполнено $A e_{n}=\alpha_{n} e_{n}$ для всех $n \in \mathbb{Z}$. Тогда

$$
S(h) A S(-h) e_{n}=S(h) A\left(e^{-i n h} e_{n}\right)=e^{-i n h} S(h) A e_{n}=e^{-i n h} S(h)\left(\alpha_{n} e_{n}\right)=e^{-i n h} \alpha_{n} S(h) e_{n}=\alpha_{n} e_{n}=A e_{n} .
$$

Соотношение выше останется справедливым, если заменить $e_{n}$ элементом $\Psi_{m} x$ для произвольного $x \in X$. Ввиду сильной сходимости последовательности $\left\{\Psi_{m}\right\}$ к тождественному оператору для всех $x \in X$ справедливо соотношение $S(h) A S(-h) x=A x$, т. е. оператор $A$ перестановочен со сдвигом.

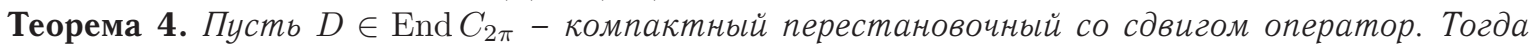
D $и \Psi_{n}$ перестановочны $и\left\|\Psi_{n} D-D\right\| \rightarrow 0$ при $n \rightarrow \infty$ в равномерной операторной топологии.

Доказательство. Поскольку оператор $D$ перестановочен со сдвигом, из леммы 5 вытекает его перестановочность с аппроксимативной единицей $\Psi_{n}$.

Для произвольного $x \in B(0,1)$ оценим величину $\left\|D \Psi_{n} x-D x\right\|=\left\|\Psi_{n} D x-D x\right\|$. Пусть $y=D x$. Фиксируем произвольное $\varepsilon>0$ и в силу компактности оператора $D$ построим конечную $\varepsilon / 3$-сеть $D B_{\varepsilon / 3}$ образа единичного шара $D(B(0,1))$. Для рассмотренного у выберем элемент сети $y_{0} \in D B_{\varepsilon / 3}$ такой, что $\left\|y-y_{0}\right\|<\varepsilon / 3$. Поскольку множество $D B_{\varepsilon / 3}$ конечно, найдем такое $n_{0} \in \mathbb{N}$, что для всех $n>n_{0}$ и для любого $y_{0} \in D B_{\delta / 3}$ выполнялось $\left\|\Psi_{n} y_{0}-y_{0}\right\|<\varepsilon / 3$. Тогда

$$
\left\|\Psi_{n} D x-D x\right\| \leq\left\|\Psi_{n}\left(y-y_{0}\right)\right\|+\left\|\Psi_{n} y_{0}-y_{0}\right\|+\left\|y_{0}-y\right\| \leq \varepsilon / 3\left(\left\|\Psi_{n}\right\|+1\right)+\left\|\Psi_{n} y_{0}-y_{0}\right\|<\varepsilon .
$$

Данная оценка не зависит от конкретного $x \in B(0,1)$, поскольку $n_{0}$ выбирается только по $\delta / 3$-сети, зависящей, в свою очередь, только от $\delta$ и оператора $D$.

Теорема 5. Пусть D- компактный перестановочный со сәвигом оператор из пространства End $C_{2 \pi}(\mathbb{R}, \mathbb{C})$, тогда он имеет вид $(D x)(t)=\int_{0}^{2 \pi} f(t-s) x(s) d s$, где $f \in L_{2 \pi}^{1}(\mathbb{R}, \mathbb{C})$ ( т. е. является оператором свертки с суммируемой функцией).

Доказательство. Покажем, что $\Psi_{n} D x=f_{n} * x$ для всех $x \in C_{2 \pi}(\mathbb{R}, \mathbb{C})$.

Рассмотрим данный оператор на функциях $e_{k}$. В силу перестановочности со сдвигом согласно лемме 5 получаем, что $D e_{k}=\alpha_{k} e_{k}, k \in \mathbb{Z}$, причем $\alpha_{k} \rightarrow 0$ при $k \rightarrow \infty$ в силу компактности оператора $D$. Тогда

$$
D \Psi_{n} e_{k}=D\left(1-\frac{|k|}{n}\right) e_{k}=\left(1-\frac{|k|}{n}\right) D e_{k}=\left(1-\frac{|k|}{n}\right) \alpha_{k} e_{k}
$$

С другой стороны,

$$
\left(f_{n} * e_{k}\right)(t)=\int_{0}^{2 \pi} f_{n}(\tau) e^{i k(t-\tau)} d \tau=2 \pi \widehat{f}_{n}(k) e_{k} .
$$

Отсюда следует, что $\widehat{f}_{n}(k)=\frac{\alpha_{k}}{2 \pi}(1-|k| / n),|k| \leq n$. Тогда $\Psi_{n} D e_{k}=f_{n} * e_{k}, k \in \mathbb{Z}$, где $f_{n}$ имеют вид

$$
f_{n}(t)=\frac{1}{2 \pi} \sum_{|k| \leq n}\left(1-\frac{|k|}{n}\right) \alpha_{k} e_{k}
$$

А значит, и для всех $x \in C_{2 \pi}(\mathbb{R}, \mathbb{C}) \quad \Psi_{n} D x=f_{n} * x$, причем доказано, что $\left\|\Psi_{n} D\right\|=\left\|f_{n}\right\|_{1}^{2 \pi}$, где $\|\cdot\|_{1}^{2 \pi}-$ норма в $L_{2 \pi}^{1}(\mathbb{R})$.

Покажем, что последовательность $\left\{f_{n}\right\}$ сходится. В силу того что $\left(D \Psi_{n}-D \Psi_{n+m}\right) x=$ $=\left(f_{n}-f_{n+m}\right) * x \quad$ для всех $x \in C_{2 \pi}(\mathbb{R}, \mathbb{C})$, получаем, что $\left\|D \Psi_{n}-D \Psi_{n+m}\right\|=\left\|f_{n}-f_{n+m}\right\|_{1}^{2 \pi}$, а 
из сходимости последовательности $D \Psi_{n}$ к оператору $D$ по норме пространства End $C_{2 \pi}(\mathbb{R}, \mathbb{C})($ теорема 4) следует ее фундаментальность. Тогда последовательность $\left\{f_{n}\right\}$ также будет фундаментальной, что в силу полноты пространства $L_{2 \pi}^{1}(\mathbb{R})$ означает ее сходимость.

Обозначим $f=\lim _{n \rightarrow \infty} f_{n}$. Тогда $D x=f * x, f \in L_{2 \pi}^{1}(\mathbb{R})$, т. е. $D-$ интегральный оператор вида $(D x)(t)=\int_{0}^{2 \pi} f(t-s) x(s) d s$.

Вернемся к рассмотрению оператора $A \in \operatorname{End}_{p} C_{2 \pi}(\mathbb{R}, \mathbb{C})$, где $p$ - один из символов $1, \alpha, \gamma$, определяемого формулой $A=a I+K$, где $a \in \mathbb{C}, a \neq 0$, а $K-$ рассмотренный ранее компактный интегральный оператор. Как было показано, его обратный имеет вид $B=A^{-1}=\frac{1}{a} I+\widetilde{K}$, где $\widetilde{K}-$ компактный оператор с абсолютно сходящимся рядом Фурье:

$$
\widetilde{K}=\sum_{n \in \mathbb{Z}} \widetilde{K}_{n}
$$

В силу компактности оператора $\widetilde{K}$ его коэффициенты Фурье $\widetilde{K}_{n}$ также компактны. Тогда операторы $\widetilde{K}_{n} E_{-n}$ компактны и перестановочны со сдвигом (в силу леммы 4). По доказанному в теореме 5 операторы $\widetilde{K}_{n} E_{-n}$ являются интегральными и имеют следующий вид:

$$
\left(\widetilde{K_{n}} E_{-n} x\right)(t)=\int_{0}^{2 \pi} \widetilde{\mathscr{K}_{n}}(t-s) x(s) d s
$$

где функции $\widetilde{\mathscr{K}_{n}} \in L_{2 \pi}^{1}(\mathbb{R}, \mathbb{C})$. Тогда оператор $\widetilde{K_{n}}$ представим в виде

$$
\left(\widetilde{K_{n}} x\right)(t)=\left(\widetilde{K_{n}} E_{-n} E_{n} x\right)(t)=\int_{0}^{2 \pi} \widetilde{\mathscr{K}_{n}}(t-s) e^{i n s} x(s) d s .
$$

Заметим, что коэффициенты Фурье операторов $K$ и $\widetilde{K}$ имеют сходную структуру, то есть каждый из операторов является произведением интегрального оператора с ядром, зависящим от разности аргументов, и оператора $E_{n}$

$$
\left(K_{n} x\right)(\tau)=\int_{0}^{2 \pi} \mathscr{K}_{n}^{0}(\tau-v) e^{i n v} x(v) d v, \quad\left(\widetilde{K_{n}} x\right)(t)=\int_{0}^{2 \pi} \widetilde{\mathscr{K}_{n}}(t-s) e^{i n s} x(s) d s .
$$

Кроме того, $\left\|\widetilde{K_{n}} E_{-n}\right\|=\left\|\widetilde{K_{n}}\right\|=\left\|\widetilde{\mathscr{K}_{n}}\right\|_{1}$. Тогда в силу абсолютной сходимости ряда Фурье оператора $\widetilde{K}$ ряд $\sum_{n \in \mathbb{Z}} \widetilde{\mathscr{K}}_{n}(t-s) e^{i n s}$ также абсолютно сходится. Обозначим

$$
\widetilde{\mathscr{K}}(t, s)=\sum_{n \in \mathbb{Z}} \widetilde{\mathscr{K}_{n}}(t-s) e^{i n s}
$$

С учетом данного обозначения для всех $x \in C_{2 \pi}(\mathbb{R}, \mathbb{C})$ имеем:

$$
\begin{aligned}
& (\widetilde{K} x)(t)=\left(\sum_{n \in \mathbb{Z}} \widetilde{K}_{n} x\right)(t)=\sum_{n \in \mathbb{Z}} \int_{0}^{2 \pi} \widetilde{\mathscr{K}}_{n}(t-s) e^{i n s} x(s) d s= \\
& =\int_{0}^{2 \pi}\left(\sum_{n \in \mathbb{Z}} \widetilde{\mathscr{K}}_{n}(t-s) e^{i n s}\right) x(s) d s=\int_{0}^{2 \pi} \widetilde{\mathscr{K}}(t, s) x(s) d s .
\end{aligned}
$$

Исследуем функцию $\widetilde{\mathscr{K}}$ и покажем, что она обладает теми же свойствами, что и ядро оператора $K$. Так как функции $\widetilde{\mathscr{K}_{n}} \in L_{2 \pi}^{1}(\mathbb{R})$ и ряд (3) абсолютно сходится, то $\widetilde{\mathscr{K}}(t, \cdot) \in L_{2 \pi}^{1}(\mathbb{R}, \mathbb{C})$. Непосредственно из определения функции $\widetilde{\mathscr{K}}$ по формуле (3) следует, что $\widetilde{\mathscr{K}}(t+2 \pi, s+2 \pi)=\widetilde{\mathscr{K}}(t, s)$ для всех $t, s \in \mathbb{R}$ и $\widetilde{\mathscr{K}}(t, \cdot) \in L_{2 \pi}^{1}(\mathbb{R}, \mathbb{C})$.

Из соотношения $\widetilde{\mathscr{K}_{n}}(t-s)=S(t) \widetilde{\mathscr{K}_{n}}(-s)$ выведем непрерывность функции $\widetilde{\mathscr{K}}(\cdot, s)$ по норме пространства $L_{2 \pi}^{1}(\mathbb{R}, \mathbb{C})$. Таким образом, доказана следующая теорема, являющаяся основным результатом работы.

Теорема 6. Пусть оператор $A \in \operatorname{Int}_{p} C_{2 \pi}(\mathbb{R}, \mathbb{C})(p-$ один из символов $1, \alpha, \gamma)$ имеет вид (1) и является непрерывно обратимым, тогда обратный оператор имеет вид

$$
A^{-1}=\frac{1}{a} I+\widetilde{K}
$$


где оператор $\widetilde{K}$ является компактным интегральным оператором вида (4) с ядром $\widetilde{\mathscr{K}}$, удовлетворяющим свойствам

1) $\widetilde{\mathscr{K}}(\tau+2 \pi, u+2 \pi)=\widetilde{\mathscr{K}}(\tau, u)$ для всех $\tau, u \in \mathbb{R}$;

2) $\widetilde{\kappa}(\tau) \in L_{2 \pi}^{1}(\mathbb{R}, \mathbb{C})$, где $(\widetilde{\kappa}(\tau))(u)=\widetilde{\mathscr{K}}(\tau, u)$ для всех $\tau, u \in \mathbb{R}$;

3) $\widetilde{\kappa} \in C_{2 \pi}\left(\mathbb{R}, L_{2 \pi}^{1}(\mathbb{R}, \mathbb{C})\right.$ (функция $\widetilde{\kappa}$ непрерывна по норме пространства $L_{2 \pi}^{1}(\mathbb{R}, \mathbb{C})$ ).

В терминах наполненности теорему 6 можно сформулировать следующим образом.

Теорема 7. Подалгебры $\operatorname{Int}_{1} C_{2 \pi}(\mathbb{R}, \mathbb{C}), \operatorname{Int}_{\alpha} C_{2 \pi}(\mathbb{R}, \mathbb{C}), \operatorname{Int}_{\gamma} C_{2 \pi}(\mathbb{R}, \mathbb{C})$ наполнены в алгебре End $C_{2 \pi}(\mathbb{R}, \mathbb{C})$.

Замечание 1. Наполненность некоторых других подалгебр, порожденных интегральными операторами, рассмотрена В. Г. Курбатовым в работе [10]. В вышеуказанной статье была исследована алгебра $\mathscr{J}\left(L_{p}(G, X)\right)$ интегральных операторов $J \in \operatorname{End} L_{p}(G, X)$, где $L_{p}(G, X), 1 \leq p \leq \infty,-$ пространства классов совпадающих локально почти всюду относильно меры Хаара измеримых функций $f: G \rightarrow X$, $G$ - компактно-порожденная группа, изоморфная группе $\mathbb{R}^{n} \times \mathbb{Z}^{m} \times \mathbb{K}$, где $n, m \in \mathbb{N}, \mathbb{K}$ - компактная абелева группа, $X$ - конечномерное банахово пространство. Операторы $J \in \mathscr{J}\left(L_{p}(G, X)\right)$ имеют вид

$$
(J x)(t)=\int_{G} N(t, s) x(s) d s,
$$

(интегрирование ведется по мере Хаара), где ядро $N$ измеримо и при некоторых $M<\infty$ и $\gamma>0$ удовлетворяет оценке

$$
\|N(t, s)\| \leq M e^{-\gamma|t-s|} .
$$

Для операторов из $\mathscr{J}\left(L_{p}(G, X)\right)$ справедлива следующая теорема.

Теорема 8. Пусть оператор $I+J$, где $J \in \mathbb{J}\left(L_{p}(G, X)\right)$, обратим в $L_{p}(G, X)$, тогда обратный оператор имеет вид $(I+J)^{-1}=I+J_{1}$, где $J_{1} \in \mathbb{J}\left(L_{p}(G, X)\right)$.

Замечание 2. Отметим, что теорема 3 используется в статье И. И. Струковой [11] для доказательства аналога теоремы Винера для периодических на бесконечности функций.

Работа выполнена при финансовой поддержке РФФИ (проект 13-01-00378).

\section{Библиограсрический список}

1. Наймарк М. А. Нормированные кольца. М. : Наука, 1968. 664 c.

2. Бурбаки Н. Спектральная теория. М. : Мир, 1972. 183 c.

3. Bochner S., Fillips R. S. Absolutely convergent Fourier expansion for non-commutative normed rings // Ann. of Math. 1942. № 3. Р. 409-418.

4. Баскаков А. Г. Теорема Винера и асимптотические оценки элементов обратных матриц // Функц. анализ и его прил. 1990. Т. 24, № 3. С. 64-65.

5. Баскаков А. Г. Абстрактный гармонический анализ и асимптотические оценки элементов обратных матриц // Мат. заметки. 1992. Т. 52, № 2. С. 17-26.

6. Баскаков А. Г. О спектральных свойствах некоторых классов линейных операторов // Функц. анализ и его прил. 1995. Т. 29, № 2. С. 61-64.

7. Баскаков А. Г. Асимптотические оценки элементов матриц обратных операторов и гармонический анализ // Сиб. мат. журн. 1997. Т. 38, № 1. С. 14-28.

8. Баскаков А. Г. Оценки элементов обратных матриц и спектральный анализ линейных операторов // Изв. РАН. Сер. мат. 1997. Т. 61, № 6. С. 3-26.

9. Баскаков А. Г. Гармонический анализ линейных операторов. Воронеж : Изд-во Воронеж. гос. ун-та, 1987. 165 c.

10. Курбатов В. Г. Об алгебрах разностных и интегральных операторов // Функц. анализ и его прил. 1990. Т. 24, № 2. С. 98-99.

11. Струкова И. И. Теорема Винера для периодических на бесконечности функций // Изв. Сарат. ун-та. Нов. сер. Сер. Математика. Механика. Информатика. 2012. Т. 12, вып. 4. С. 34-41.

\section{Structure of the Inverse for the Integral Operator of Special Kind}

\section{E. Strukov}

Voronezh State University, Russia, 394006, Voronezh, Universitetskaya pl., 1, sv.post.of.chaos@gmail.com

Algebra (with identity) generated by integral operators on the spaces of continuous periodic functions is considered. This algebra is proved to be an inverse-closed subalgebra in the algebra of all bounded linear operators.

Key words: Banach space, integral operator, Bochner-Phillips theorem, Fourier series of an operator, inverse-closedness of a subalgebra, Wiener's pair of algebras. 


\section{References}

1. Naimark M. A. Normirovannye kol'tsa [Normed Rings]. Moscow, Nauka Publ., 1968, 664 p. (in Russian). 2. Burbaki N. Spektral'naya teoriya [Spectral theory]. Moscow, Mir, 1972, 183 p. (in Russian).

3. Bochner S., Fillips R. S. Absolutely convergent Fourier expansion for non-commutative normed rings. Ann. of Math., 1942, no. 3, pp. 409-418.

4. Baskakov A. G. Wiener's theorem and the asymptotic estimates of the elements of inverse matrices. Functional Analysis and Its Applications, 1990, vol. 24, no. 3, pp. 222-224.

5. Baskakov A. G. Absteact haemonic analysis and asymptotic estimates of elements of inverse matrices. Math. Notes, 1992, vol. 52, iss. 2, pp. 764-771.

6. Baskakov A. G. On Spectral Properties of Some Classes of Linear Operators. Functional Analysis and Its Applications, 1995, vol. 29, no. 2, pp. 121-123.
7. Baskakov A. G. Asymptotic estimates for elements of matrices of inverse operators, and harmonic analysis. Siberian Math. J., 1997, vol. 38, iss. 1, pp. 10-22.

8. Baskakov A. G. Estimates for the entries of inverse matrices and the spectral analysis of linear operators. Izvestiya: Mathematics, 1997, vol. 61, no. 6, pp. 11131135. DOI: $10.4213 / \mathrm{im} 164$.

9. Baskakov A. G. Garmonicheskii analiz lineinykh operatorov [Harmonic analysis of linear operators]. Voronezh, Voronezh Univ. Press, 1987, 165 p. (in Russian).

10. Kurbatov V. G. Algebras of difference and integral operators. Functional Analysis and Its Applications, 1990, vol. 24, no. 2, pp. 156-158.

11. Strukova I. I. Wiener's theorem for periodic at infinity functions. Izv. Sarat. Univ. N.S. Ser. Math. Mech. Inform., 2012, vol. 12, iss. 4, pp. 34-41 (in Russian).

УДК 517.547.2

\title{
К ПРОБЛЕМЕ ЛЕОНТЬЕВА О ЦЕЛЫХ ФУНКЦИЯХ ВПОЛНЕ РЕГУЛЯРНОГО РОСТА
}

\section{В. Б. Шерстюков}

\begin{abstract}
Кандидат фризико-математических наук, доцент кафедры высшей математики, Национальный исследовательский ядерный университет «МИФИ», Москва, shervb73@gmail.com

Рассматривается произвольная целая функция экспоненциального типа, все нули которой просты и образуют последовательность с нулевым индексом конденсации. На множестве нулей такой функции ее производная растет в определенном смысле максимально быстро. Требуется выяснить, будет ли исходная фрункция обладать полной регулярностью роста. Эта задача, возникшая в теории представления аналитических функций рядами экспонент, была поставлена А. Ф. Леонтьевым более сорока лет назад и пока не решена. В настоящей работе показано, что означенная проблема решается положительно, если фрункция «не слишком мала» на некоторой прямой.
\end{abstract}

Ключевые слова: проблема Леонтьева, фрункция вполне регулярного роста, индекс конденсации.

\section{1. ПОСТАНОВКА ЗАДАЧИ}

Изучая вопросы, связанные с представлением аналитических функций рядами экспонент, А. Ф. Леонтьев (1972 г.) поставил следующую задачу [1, замечание на с. 1291]. Пусть $L(\lambda)-$ целая функция экспоненциального типа (ЦФЭТ) с последовательностью простых (всех) нулей $\Lambda=\left(\lambda_{k}\right)_{k=1}^{\infty}$ и индикатором

$$
h_{L}(\theta) \equiv \varlimsup_{r \rightarrow+\infty} \frac{\ln \left|L\left(r e^{i \theta}\right)\right|}{r}, \quad \theta \in[0,2 \pi],
$$

удовлетворяющая условию

$$
\lim _{k \rightarrow \infty}\left\{\frac{1}{\left|\lambda_{k}\right|} \ln \left|L^{\prime}\left(\lambda_{k}\right)\right|-h_{L}\left(\arg \lambda_{k}\right)\right\}=0 .
$$

Нужно выяснить, является ли $L(\lambda)$ функцией вполне регулярного роста (ВРP).

Последнее свойство в соответствии с общим определением [2, гл. III] равносильно существованию равномерного по $\theta \in[0,2 \pi]$ предела

$$
\lim _{\substack{r \rightarrow+\infty \\ r \notin E}} \frac{\ln \left|L\left(r e^{i \theta}\right)\right|}{r}=h_{L}(\theta) .
$$

Здесь $E-$ некоторое множество положительных чисел нулевой относительной меры, т. е. такое, что множество $E \cap[0, r]$ измеримо (по Лебегу) при каждом $r>0$ и его мера есть $o(r)$ при $r \rightarrow+\infty$. 